\title{
A fast method for solving a linear model of one-dimensional blood flow in a viscoelastic arterial tree
}

\author{
Ivan Korade, Zdravko Virag and Severino Krizmanić
}

\begin{abstract}
For the purpose of optimization of the whole arterial tree, a fast method for solving of one-dimensional model of blood flow is required. A semi-analytic transmission line method for solving a linearized one-dimensional model of blood flow in an arterial tree with viscoelastic walls is proposed. The transmission line method that solves the linearized model in the frequency domain and the method of characteristics that solves either linearized or non-linear one-dimensional models in the time domain are compared regarding accuracy and computational time. For this purpose, the benchmark problem of a 37-artery network with available experimental data is used. In the case of the linearized model, the results from the transmission line method and the method of characteristics are practically the same. The difference between the transmission line method solution of the linearized model and the method of characteristics solution of the non-linear model is much smaller than the error of either method of characteristics or transmission line method numerical solutions with respect to the experimental data. For typical applications, the transmission line method is at least two orders of magnitude faster than the method of characteristics.
\end{abstract}

\section{Keywords \\ Voigt model, non-linear versus linearized model, frequency versus time domain, computational time, cardiovascular sys- tem mechanics}

Date received: 2 August 2016; accepted: 20 December 2016

\section{Introduction}

Lots of research has been devoted to the description of the human arterial network by simple models. The simplest are lumped (Windkessel) models, which contain a small number of parameters and describe the heart afterload properly. ${ }^{1}$ One-dimensional (1D) models are popular in simulations of the pressure and flow rate wave traveling along the arterial tree. In general, 1D models are non-linear and they can be solved in the time domain by various methods: finite difference, ${ }^{2-4}$ finite volume, ${ }^{5,6}$ finite element, ${ }^{7-10}$ or the method of characteristics (MOC) ${ }^{11-14}$ It is also convenient to linearize 1D models and to solve them in the frequency domain by the transmission line method (TLM). There are two approaches in the TLMs. In the first approach, the inertance, resistance and the compliance of each arterial segment are concentrated into LRC elements of an electrical analogue scheme, which is equivalent to a Windkessel model with a number of compartements. ${ }^{15,16}$ In the second approach, the momentum and constitutive equations (from 1D models) are integrated over the segment length. In some TLMs, ${ }^{17-19}$ the Womersley solution of pulsatile flow in an elastic pipe is used, and in some TLMs, the momentum equation is integrated either numerically ${ }^{20}$ or analytically. ${ }^{21-24}$ In most of these methods, an elastic arterial wall is assumed, and in the methods based on the Womersley solution, the wall viscosity is taken into account through the modification of the characteristic impedance and the propagation constant. ${ }^{17-19}$ In case of a tree-like structure of the arterial network, the solution strategy in TLMs is straightforward: in the first step, the impedance is calculated at network nodes, and in the second step, phasors of pressure and flow rate are calculated. The main drawback of a TLM is in solving

Faculty of Mechanical Engineering and Naval Architecture, University of Zagreb, Zagreb, Croatia

\section{Corresponding author:}

Ivan Korade, Faculty of Mechanical Engineering and Naval Architecture, University of Zagreb, Ivana Lučića 5, 10000 Zagreb, Croatia.

Email: ivan.korade@fsb.hr 
a reduced mathematical model in which a non-linear convection term is neglected, and consequently, the solution accuracy is reduced. All time domain methods are more computationally demanding because they require solving a discretized system of linear algebraic equations in each integration time step and because of the need to integrate model equations over several heart periods to diminish the influence of initial conditions. In problems including optimization of the whole arterial tree, a fast method for solving the 1D model of blood flow is needed, and a TLM can be a good candidate. The question is how much faster is a TLM than a time domain method, and whether the accuracy of the linearized model is acceptable in typical problems.

The goals of this article are as follows:

1. To define a TLM for solving the linearized form of the 1D model of blood flow in an arterial tree. With respect to existing methods, the difference is in the wall viscoelasticity that is modeled by the Voigt model in the same way as in the non-linear model. Boundary conditions can be prescribed at any node of the arterial tree, using the inertial fourelement Windkessel model;

2. To verify the developed method by comparison of the solutions of the linearized model from the TLM and in-house developed $\mathrm{MOC},{ }^{14}$ which solves the same model in the time domain;

3. To validate the TLM in a benchmark problem with available experimental data. ${ }^{7,25,26}$ Results from the linearized model (obtained by the TLM) will be compared with the measurements and with the numerical results from the non-linear model (obtained by the MOC);

4. To compare computational times needed to obtain the solution by the TLM and the MOC.

\section{Method}

\section{ID model of blood flow}

A widely used 1D model of blood flow in a large artery element with an impermeable viscoelastic wall ${ }^{25}$ reads

$$
\begin{aligned}
& \frac{\partial A}{\partial t}+\frac{\partial Q}{\partial x}=0 \\
& \frac{\partial Q}{\partial t}+\frac{A}{\rho} \frac{\partial p}{\partial x}+\frac{\partial(Q v)}{\partial x}=-f Q
\end{aligned}
$$

where $x$ and $t$ are the space and the time coordinate, respectively, $A$ is the cross-sectional area $A=\left(D^{2} \pi\right) / 4$, $Q$ is the volume flow rate, $v=Q / A$ is the averaged axial velocity, $p$ is the transmural pressure, $\rho$ is the blood density and $f$ is the friction coefficient, defined by

$$
f=\frac{2(\zeta+2) \pi \mu}{\rho A}
$$

where $\mu$ is the blood viscosity and $\zeta$ is the order of the axial velocity profile. ${ }^{27}$ The constitutive equation for a viscoelastic wall reads

$$
p=p_{\mathrm{e}}+\eta \frac{\partial A}{\partial t}=p_{0}+\frac{1}{C_{D}}\left(\sqrt{A}-\sqrt{A_{0}}\right)+\eta \frac{\partial A}{\partial t}
$$

where $p_{\mathrm{e}}$ is the elastic part of the transmural pressure $p, \eta$ is the viscous resistance of the wall, $A_{0}$ is the constant cross-sectional area at constant pressure $p_{0}$, $C_{D}=3 A_{0} /(4 \sqrt{\pi} E \delta), E$ is Young's modulus and $\delta$ is the wall thickness, $\eta=2 \sqrt{\pi} \varphi \delta /\left(3 A_{0} \sqrt{A}\right)$, where $\varphi$ is the wall viscosity. The areal compliance $C=\mathrm{d} A / \mathrm{d} p_{\mathrm{e}}$ is related to $C_{D}$ by

$$
C=2 C_{D} \sqrt{A}
$$

The speed of sound is by definition

$$
c=\sqrt{\frac{A}{\rho C}}=\sqrt{\frac{1}{2 \rho C_{D}}} A^{\frac{1}{4}}
$$

When $E$ and $\varphi$ are constants, the retardation time constant $\tau$ takes the form

$$
\tau=\eta C=\frac{\varphi}{E}
$$

Equation (2) is non-linear due to a convection term (the third term at the left-hand side) and pressure dependent $A$ (see the coefficient in the second term at the left-hand side).

\section{Linearized mathematical model}

In the linearized mathematical model, the convection term is neglected and all coefficients are approximated by constants (using their values at average pressure). For each arterial segment, the average pressure is

$$
p_{\text {av }}=\frac{1}{T} \int_{0}^{T} p \mathrm{~d} t
$$

where $T$ is the heart period. Averaged cross-sectional area is approximated using equation (4), and by neglecting viscous part of pressure, in the form

$$
A_{\mathrm{av}}=\left(\sqrt{A_{0}}+C_{D}\left(p_{\mathrm{av}}-p_{0}\right)\right)^{2}
$$

$C_{\mathrm{av}}$ is calculated from equation (5) using $A_{\mathrm{av}}$, and $\eta_{\mathrm{av}}$ from equation (7) using $C_{\mathrm{av}}$. In the linearized model, equation (2) takes the form

$$
\frac{\partial Q}{\partial t}+\frac{A_{\mathrm{av}}}{\rho} \frac{\partial p}{\partial x}=-f Q
$$

and equation (4) is linearized in the form

$$
p=p_{\mathrm{av}}+\frac{1}{C_{\mathrm{av}}}\left(A-A_{\mathrm{av}}\right)+\eta_{\mathrm{av}} \frac{\partial A}{\partial t}
$$

\section{Boundary conditions}

When considering an arterial tree, boundary conditions at the network inlet and outlet nodes should be 


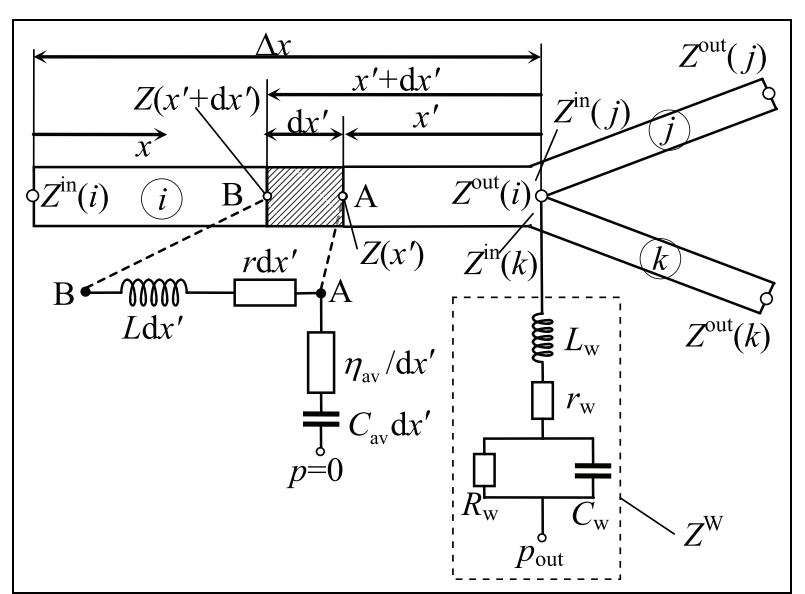

Figure I. Electrical analogue scheme of a part of an arterial network consisting of three elements: $i, j$ and $k$. The node outflow condition is prescribed by the inertial four-element Windkessel model. An infinitesimal part of arterial element is modeled by inertance $L d x^{\prime}$ and resistance $r d x^{\prime}$, while the constitutive equation of the arterial wall is modeled by resistance $\eta_{\mathrm{av}} / \mathrm{d} x^{\prime}$ and capacitance $C_{\mathrm{av}} \mathrm{dx}$.

prescribed. Here, at the inlet node, the flow rate is prescribed. At the outlet nodes, the inertial four-element Windkessel model, as depicted in Figure 1, prescribes the outflow boundary condition. It is assumed that the constant outflow backpressure $p_{\text {out }}$ (the pressure at which the flow to the periphery ceases) can be different from the extramural pressure which is assumed to be zero. At junction nodes, we assume that the static pressure is preserved.

\section{TLM for solving linearized model}

We consider blood flow in an arterial network of treelike structure. At any cross-section of the network, we can decompose a periodic pressure and flow rate signal into a Fourier series

$$
\begin{aligned}
p^{\prime}(t)= & p(t)-p_{\text {out }}=C_{0}^{p}+\sum_{n=1}^{N} S_{n}^{p} \sin \left(n \omega_{0} t\right) \\
& +C_{n}^{p} \cos \left(n \omega_{0} t\right) \\
Q(t)= & C_{0}^{Q}+\sum_{n=1}^{N} S_{n}^{Q} \sin \left(n \omega_{0} t\right)+C_{n}^{Q} \cos \left(n \omega_{0} t\right)
\end{aligned}
$$

and define the impedance $Z$ as

$$
Z_{n}=Z\left(n \omega_{0}\right)=\frac{\hat{p}_{n}^{\prime}\left(n \omega_{0}\right)}{\hat{Q}_{n}\left(n \omega_{0}\right)} \quad n=0, N
$$

where $n$ is the harmonic number, $N$ is the total number of harmonics, $\omega_{0}$ is the fundamental frequency $\omega_{0}=2 \pi / T, \hat{p}_{n}^{\prime}=S_{n}^{p}+\mathrm{i} C_{n}^{p}$ and $\hat{Q}_{n}=S_{n}^{Q}+\mathrm{i} C_{n}^{Q}$ denote phasors of pressure and flow rate, respectively, and $\mathrm{i}=\sqrt{-1}$.

Figure 1 shows a part of an arterial network: segment $i$ branching into segments $j$ and $k$. Two nodes bound each segment and the node number at the element outlet is equal to the element number. The impedances $Z^{\text {in }}$ and $Z^{\text {out }}$ are calculated at the each element's inlet and outlet, respectively. Outlet boundary condition can be defined at any node and it is modeled by the inertial four-element Windkessel model (containing inertance $L_{\mathrm{W}}$, resistances $r_{\mathrm{W}}$ and $R_{\mathrm{W}}$ and compliance $C_{\mathrm{W}}$ ), that is, by the impedance $Z^{\mathrm{W}}$ defined by

$$
Z_{n}^{\mathrm{W}}=r_{\mathrm{W}}+\mathrm{i} \omega_{n} L_{\mathrm{W}}+\frac{R_{\mathrm{W}}}{1+\mathrm{i} \omega_{n} C_{\mathrm{W}} R_{\mathrm{W}}}
$$

where $\omega_{n}=n \omega_{0}$. At the outlet of ith element in Figure 1, the impedance is defined by

$$
\frac{1}{Z_{n}^{\text {out }}(i)}=\frac{1}{Z_{n}^{\mathrm{W}}}+\frac{1}{Z_{n}^{\text {out }}(j)}+\frac{1}{Z_{n}^{\text {out }}(k)}
$$

equation (16) holds for all harmonics (frequencies).

It follows from equation (10) that an infinitesimal length $\mathrm{d} x$ of the element is modeled by inertance $L \mathrm{~d} x^{\prime}=\rho \mathrm{d} x^{\prime} / A_{\mathrm{av}}$ in series with resistance $r \mathrm{~d} x^{\prime}=\rho f \mathrm{~d} x^{\prime} / A_{\mathrm{av}}=2(\zeta+2) \pi \mu \mathrm{d} x^{\prime} / A_{\mathrm{av}}^{2}$. According to equation (11), the viscoelasticity of the arterial wall is modeled by resistance $\eta_{\mathrm{av}} / \mathrm{d} x^{\prime}$ and capacitance $C_{\mathrm{av}} \mathrm{d} x^{\prime}$ (see Figure 1). The increase in the impedance $\mathrm{d} Z_{n}=Z_{n}\left(x^{\prime}+\mathrm{d} x^{\prime}\right)-Z_{n}\left(x^{\prime}\right)$ is defined by

$$
\begin{aligned}
& Z_{n}\left(x^{\prime}+\mathrm{d} x^{\prime}\right)=\mathrm{i} \omega_{n} \frac{\rho}{A_{\mathrm{av}}} \mathrm{d} x^{\prime}+r \mathrm{~d} x^{\prime} \\
& +\frac{Z_{n}\left(x^{\prime}\right)\left(1+\mathrm{i} \omega_{n} C_{\mathrm{av}} \eta_{\mathrm{av}}\right)}{\left(1+\mathrm{i} \omega_{n} C_{\mathrm{av}} \eta_{\mathrm{av}}\right)+\mathrm{i} \omega_{n} C_{\mathrm{av}} Z_{n}\left(x^{\prime}\right) \mathrm{d} x^{\prime}}
\end{aligned}
$$

By neglecting terms with $\mathrm{d} x^{\prime 2}$, the previous equation can be rearranged into

$$
\frac{\mathrm{d} Z_{n}}{a^{2} Z_{n}^{2}-Z_{0 n}^{2}}=-\mathrm{i} \omega_{n} C_{\mathrm{av}} \mathrm{d} x^{\prime}
$$

where

$$
\begin{aligned}
& Z_{0 n}=\sqrt{\frac{\rho}{A_{\mathrm{av}} C_{\mathrm{av}}}+\frac{r}{\mathrm{i} \omega_{n} C_{\mathrm{av}}}} \\
& a_{n}=\sqrt{\frac{1}{1+\mathrm{i} \omega_{n} C_{\mathrm{av}} \eta_{\mathrm{av}}}}
\end{aligned}
$$

Integration of equation (18) along $i$ th element, from the element outlet $\left(x^{\prime}=0\right.$ and $\left.Z_{n}=Z_{n}^{\text {out }}(i)\right)$ to the element inlet $\left(x^{\prime}=\Delta x\right.$ and $\left.Z_{n}=Z_{n}^{\text {in }}(i)\right)$ gives

$$
Z_{n}^{\text {in }}(i)=\frac{Z_{0 n}}{a} \frac{\left[a Z_{n}^{\text {out }}(i)+Z_{0 n}\right]+\left[a Z_{n}^{\text {out }}(i)-Z_{0 n}\right] e^{-2 \mathrm{i} \omega_{n} C_{\text {av }} Z_{0 n} a_{n} \Delta x}}{\left[a Z_{n}^{\text {out }}(i)+Z_{0 n}\right]-\left[a Z_{n}^{\text {out }}(i)-Z_{0 n}\right] e^{-2 \mathrm{i} \omega_{n} C_{\mathrm{av}} Z_{0 n} a_{n} \Delta x}}
$$


By applying equations (16) and (21) to all elements in the network (starting from the end elements and going to the inlet element), the impedance at all element inlets and outlets is calculated. The input impedance of the whole arterial tree corresponds to $Z^{\text {out }}(1)$, where index 1 denotes the inlet of the arterial tree. Using $Z^{\text {out }}(1)$, it is possible to calculate the phasor of the inlet flow rate from the prescribed inlet pressure or the phasor of the inlet pressure from the prescribed inlet flow rate.

According to Figure 1, we can state that the phasor of pressure change along $x$ is defined by

$$
\frac{\mathrm{d} \hat{p}_{n}^{\prime}}{\mathrm{d} x}=-\left(\mathrm{i} \omega_{n} L+r\right) \hat{Q}_{n}=z_{n}^{p} \hat{Q}_{n} \quad n=0, N
$$

and the change of the flow rate phasor along $x$ is defined by

$$
\frac{\mathrm{d} \hat{Q}_{n}}{\mathrm{~d} x}=-\frac{\mathrm{i} \omega_{n} C_{\mathrm{av}}}{\left(1+\mathrm{i} \omega_{n} C_{\mathrm{av}} \eta_{\mathrm{av}}\right)} \hat{p}_{n}^{\prime}=z_{n}^{Q} \hat{p}_{n}^{\prime} \quad n=0, N
$$

Equations (22) and (23) can be rearranged in the following forms:

$$
\frac{\mathrm{d}^{2} \hat{p}_{n}^{\prime}}{\mathrm{d} x^{2}}-z_{n}^{p} z_{n}^{Q} \hat{p}_{n}^{\prime}, n=0, N
$$

with initial conditions at $x=0: \quad \hat{p}_{n}^{\prime}=\hat{p}_{n}^{\prime}$ in and $\mathrm{d} \hat{p}_{n}^{\prime} / \mathrm{d} x=-z_{n}^{p} \hat{Q}_{n}^{\text {in }}$, and

$$
\frac{\mathrm{d}^{2} \hat{Q}_{n}}{\mathrm{~d} x^{2}}-z_{n}^{p} z_{n}^{Q} \hat{Q}_{n}, n=0, N
$$

with initial conditions at $x=0: \hat{Q}_{n}=\hat{Q}_{n}^{\text {in }} \quad$ and $\mathrm{d} \hat{Q}_{n} / \mathrm{d} x=-z_{n}^{Q} \hat{p}_{n}^{\text {in }}$. After analytic integration of equations (24) and (25), from the element inlet to its outlet, it follows

$$
\begin{aligned}
\hat{p}_{n}^{\text {out }}= & \frac{1}{2}\left(\hat{p}_{n}^{\text {in }}+\sqrt{\frac{z_{n}^{p}}{z_{n}^{Q}}} \hat{Q}_{n}^{\text {in }}\right) e^{-\sqrt{z_{n}^{p} z_{n}^{Q}} \Delta x} \\
& +\frac{1}{2}\left(\hat{p}_{n}^{\text {in }}-\sqrt{\frac{z_{n}^{p}}{z_{n}^{Q}}} \hat{Q}_{n}^{\text {in }}\right) e^{\sqrt{z_{n}^{p} z_{n}^{Q}} \Delta x} \\
\hat{Q}_{n}^{\text {out }}= & \frac{1}{2}\left(\hat{Q}_{n}^{\text {in }}+\sqrt{\frac{z_{n}^{Q}}{z_{n}^{p}}} \hat{p}_{n}^{\text {in }}\right) e^{-\sqrt{z_{n}^{p} z_{n}^{Q}} \Delta x} \\
& +\frac{1}{2}\left(\hat{Q}_{n}^{\text {in }}-\sqrt{\frac{z_{n}^{Q}}{z_{n}^{p}}} \hat{p}_{n}^{\text {in }}\right) e^{\sqrt{z_{n}^{p} z_{n}^{Q}} \Delta x}
\end{aligned}
$$

Equations (26) and (27) are applied to all elements, starting from the arterial tree inlet and going to peripheral elements. Once the phasors of pressure and flow rate are known, the solution in the time domain is calculated according to equations (12) and (13).

Since the average pressure at each element is not known in advance, the numerical procedure is an iterative one. When the inlet pressure is prescribed, its average value is used as an initial value for all elements. When the inlet flow rate is prescribed, the initial

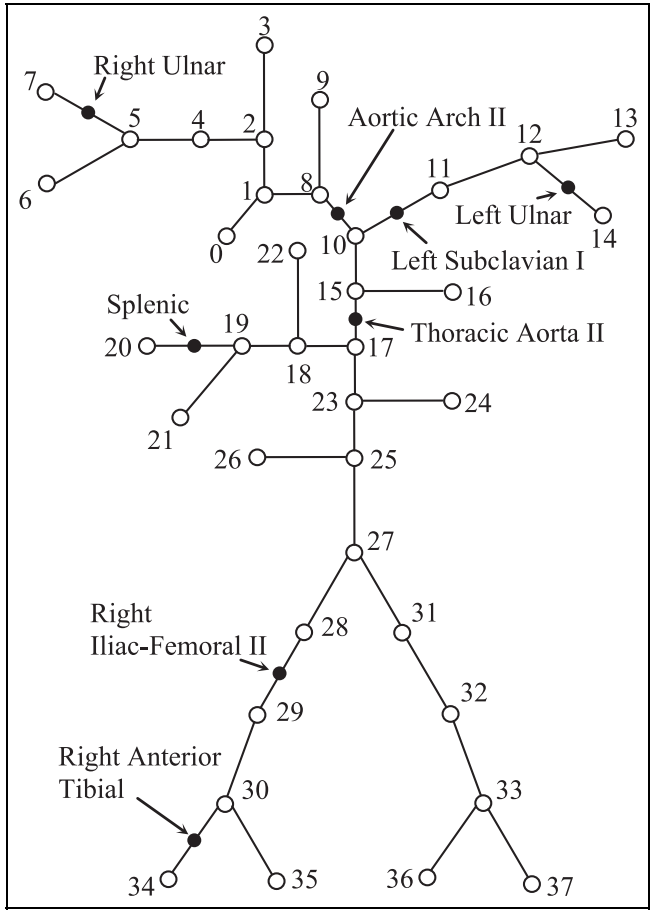

Figure 2. Scheme of a 37-segment arterial tree. The segment number is equal to the number of its end node. The node number zero denotes the arterial tree inlet where a periodic flow rate was prescribed. Basic data for all segments and outlet boundary conditions that are modeled by a single resistor are given in Table I. Filled circles denote measurement sites.

average pressure is calculated as a product of average input flow rate and total resistance $\left(R_{\mathrm{tot}}\right)$ calculated at $\omega_{n}=0$. The iterative procedure finishes when the changes in average pressure from two successive iterations at all elements fall below $1 \mathrm{~Pa}$, and for this, usually two or three iterations are needed.

\section{MOC}

Beside the described TLM, we also use the MOC, ${ }^{14}$ which solves either a non-linear model or a linearized model in time domain. When using any time domain method, there is need to prescribe the initial conditions and the integration time should be sufficiently long to achieve a periodic solution (to diminish the influence of initial conditions).

\section{Results}

The two methods were applied to a 37-artery model of an arterial tree ${ }^{25,26}$ with available experimental data. Figure 2 shows a scheme of the considered arterial tree, in which filled circles denote measurement sites with available experimental data. All data relevant to this problem are provided in the supplementary material. ${ }^{25}$ Data for all segments are given in Table 1. Some segments have a variable radius and the speed of sound, and their values at pressure $p_{0}$ at the segment inlet and outlet are indicated in Table 1 by $r_{0-\text { in }} / r_{0-\text { out }}$ and 
Table I. Data of the 37-artery network test.

\begin{tabular}{|c|c|c|c|c|c|c|}
\hline No. & Arterial segment & Length $(\mathrm{m})$ & $r_{0-\text { in }} / r_{0-\text { out }}(\mathrm{mm})$ & $c_{0-\text { in }} / c_{0-\text { out }}(\mathrm{m} / \mathrm{s})$ & $R_{\mathrm{W}}\left(\mathrm{GPa} s / \mathrm{m}^{3}\right)$ & $\begin{array}{l}\text { No. of MOC } \\
\text { elements }\end{array}$ \\
\hline I & Ascending aorta & 0.036 & $14.4 / \mid 3.0$ & $5.21 / 5.49$ & - & 8 \\
\hline 2 & Innominate & 0.028 & $11.0 / 7.29$ & $4.89 / 6.01$ & - & 6 \\
\hline 3 & Right carotid & 0.145 & $5.37 / 3.86$ & $6.35 / 7.49$ & 2.67 & 24 \\
\hline 4 & Right subclavian I & 0.218 & $4.36 / 3.34$ & $6.87 / 7.84$ & - & 34 \\
\hline 5 & Right subclavian II & 0.165 & $3.34 / 2.78$ & $6.00 / 6.58$ & - & 30 \\
\hline 6 & Right radial & 0.235 & $2.07 / 2.07$ & $7.43 / 7.43$ & 3.92 & 36 \\
\hline 7 & Right ulnar & 0.177 & $2.10 / 2.10$ & $8.81 / 8.81$ & 3.24 & 24 \\
\hline 8 & Aortic arch I & 0.021 & $13.0 / 12.5$ & $5.4 I / 5.52$ & - & 4 \\
\hline 9 & Left carotid & 0.178 & $5.58 / 3.73$ & $6.55 / 8.00$ & 3.11 & 28 \\
\hline 10 & Aortic arch II & 0.029 & $|2.5 /| 1.8$ & $4.98 / 5.12$ & - & 8 \\
\hline II & Left subclavian I & 0.227 & $4.42 / 3.39$ & $6.21 / 7.10$ & - & 40 \\
\hline 12 & Left subclavian II & 0.175 & $3.39 / 2.84$ & $6.26 / 6.84$ & - & 31 \\
\hline 13 & Left radial & 0.245 & $2.07 / 2.07$ & $8.84 / 8.84$ & 3.74 & 32 \\
\hline 14 & Left ulnar & 0.191 & $2.07 / 2.07$ & 7.77/7.77 & 3.77 & 28 \\
\hline 15 & Thoracic aorta I & 0.056 & $11.8 / 11.0$ & $5.29 / 5.48$ & - & 12 \\
\hline 16 & Intercostals & 0.195 & $4.12 / 3.22$ & 7.07/7.99 & 2.59 & 30 \\
\hline 17 & Thoracic aorta II & 0.072 & II.0/9.26 & $4.84 / 5.26$ & - & 16 \\
\hline 18 & Celiac I & 0.038 & $3.97 / 3.97$ & $6.20 / 6.20$ & - & 7 \\
\hline 19 & Celiac II & 0.013 & $4.31 / 4.31$ & $14.9 / 14.9$ & - & 1 \\
\hline 20 & Splenic & 0.191 & $1.83 / 1.83$ & $7.24 / 7.24$ & 3.54 & 30 \\
\hline 21 & Gastric & 0.198 & $1.92 / 1.92$ & $6.73 / 6.73$ & 4.24 & 34 \\
\hline 22 & Hepatic & 0.186 & $3.31 / 2.89$ & $6.95 / 7.44$ & 3.75 & 30 \\
\hline 23 & Abdominal aorta I & 0.062 & $9.26 / 8.01$ & $5.19 / 5.59$ & - & 13 \\
\hline 24 & Left renal & 0.120 & $2.59 / 2.59$ & 7.39/7.39 & 3.46 & 19 \\
\hline 25 & Abdominal aorta II & 0.007 & $7.90 / 7.90$ & $5.83 / 5.83$ & - & 1 \\
\hline 26 & Right renal & 0.118 & $2.55 / 2.55$ & $6.95 / 6.95$ & 3.45 & 19 \\
\hline 27 & Abdominal aorta III & 0.104 & $7.80 / 5.88$ & $5.4 I / 6.24$ & - & 20 \\
\hline 28 & Right iliac-femoral I & 0.205 & $3.90 / 3.38$ & $6.47 / 6.94$ & - & 35 \\
\hline 29 & Right iliac-femoral II & 0.216 & $3.38 / 2.31$ & $5.89 / 7.13$ & - & 38 \\
\hline 30 & Right iliac-femoral III & 0.206 & $2.31 / 2.10$ & $8.04 / 8.44$ & - & 29 \\
\hline 31 & Left iliac-femoral I & 0.201 & $4.02 / 3.34$ & $6.19 / 6.79$ & - & 35 \\
\hline 32 & Left iliac-femoral II & 0.195 & $3.34 / 2.26$ & $6.11 / 7.44$ & - & 33 \\
\hline 33 & Left iliac-femoral III & 0.207 & $2.26 / 2.12$ & $6.67 / 6.89$ & - & 35 \\
\hline 34 & Right anterior tibial & 0.163 & $1.55 / 1.55$ & $8.47 / 8.47$ & 5.16 & 22 \\
\hline 35 & Right posterior tibial & 0.151 & $1.53 / 1.53$ & 7.73/7.73 & 5.65 & 22 \\
\hline 36 & Left posterior tibial & 0.149 & $1.58 / 1.58$ & $7.23 / 7.23$ & 4.59 & 24 \\
\hline 37 & Left anterior tibial & 0.126 & $1.55 / 1.55$ & $7.01 / 7.01$ & 3.16 & 21 \\
\hline
\end{tabular}

$r_{0-\text { in }} / r_{0-\text { out }}$ : segment radius at the reference pressure at the segment inlet/outlet; $c_{0-\text { in }} / c_{0-\text { out }}$ : speed of sound at the reference pressure at the segment inlet/outlet; $R_{\mathrm{W}}$ : resistance in the outlet boundary condition (see Figure I). The last column denotes the number of divisions of each segment used in the method of characteristics (MOC).

$c_{0-\text { in }} / c_{0-\text { out }}$, respectively. In the numerical procedure, segments were divided into a number of elements (number of divisions for all segments are also provided in Table 1) and $r_{0}$ and $c_{0}$ were defined at the midpoint of each element. We assumed a linear variation in the radius from the segment inlet to its outlet, and $c_{0}$ is interpolated on the assumption that $c_{0}^{2} r_{0}=K=$ const. The constant $K$ was calculated as a geometric mean from the inlet and outlet data. The total number of elements was 860 . The arterial wall is considered as viscoelastic, and the retardation time constant was $3 \mathrm{~ms}$ for all elements. Element lengths were in the range from 3.63 to $13 \mathrm{~mm}$, the integration time step was $0.2 \mathrm{~ms}$ and Courant number values were from 0.166 to 0.282 .

At the inlet node (node 0 in Figure 2), the periodic flow rate is prescribed by the Fourier series
Table 2. Model parameters of the 37-artery network.

\begin{tabular}{ll}
\hline Property & Value \\
\hline Blood density, $\rho\left(\mathrm{kg} / \mathrm{m}^{3}\right)$ & 1050 \\
Blood viscosity, $\mu(\mathrm{MPa})$ & 2.5 \\
Initial pressure, $p_{0}(\mathrm{KPa})$ & 0 \\
Retardation time constant, $\tau(\mathrm{ms})$ & 3 \\
Outflow pressure, $p_{\text {out }}(\mathrm{Pa})$ & 432.6 \\
Velocity profile order, $\zeta$ & 9 \\
\hline
\end{tabular}

containing 41 harmonics as given in Supplementary Material. ${ }^{25}$ At the end nodes of the arterial tree a single resistor models the outlet boundary conditions. The values of resistance $R_{\mathrm{W}}$ (see Figure 1) are reported in Table 1 . The used values of model parameters are specified in Table 2. 


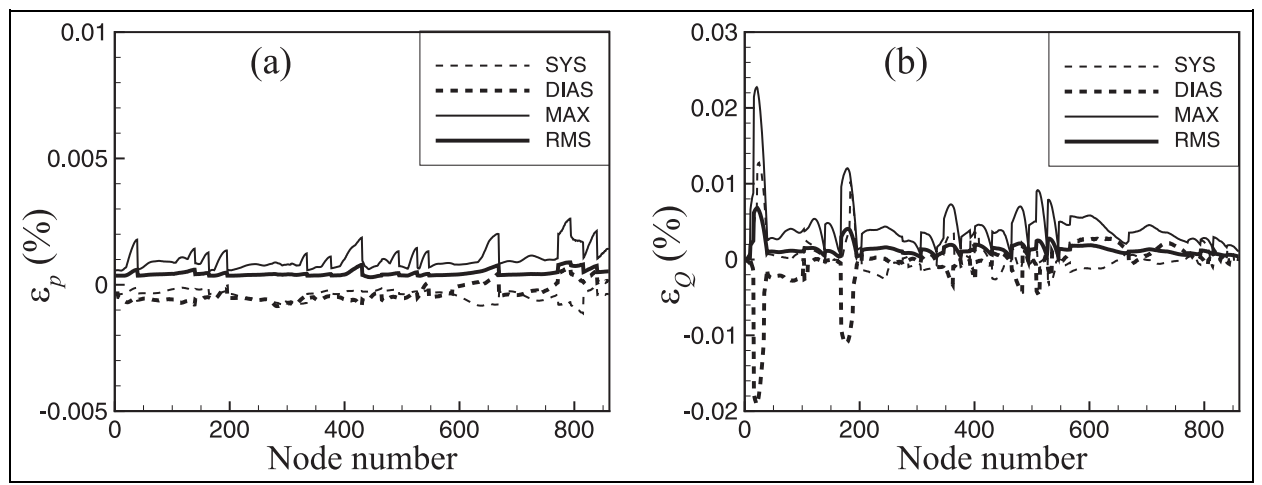

Figure 3. Relative percentage errors from the linear model obtained by the method of characteristics (MOC) with respect to the solution of transmission line method (TLM): (a) Errors in pressure: $\varepsilon_{p}^{\mathrm{RMS}}, \varepsilon_{p}^{\mathrm{SYS}}, \varepsilon_{p}^{\mathrm{DIAS}}$ and $\varepsilon_{p}^{\mathrm{MAX}}$ and (b) Errors in flow rate: $\varepsilon_{Q}^{\mathrm{RMS}}, \varepsilon_{Q}^{\mathrm{SYS}}$, $\varepsilon_{Q}^{\mathrm{DIAS}}$ and $\varepsilon_{Q}^{\mathrm{MAX}}$. Errors defined by equations (28)-(35).

For the purpose of comparison of two solutions, the following errors are defined

$$
\begin{aligned}
& \varepsilon_{p}^{\mathrm{RMS}}=\sqrt{\frac{1}{N_{t}} \sum_{i=1}^{N_{t}}\left(\frac{p_{i}-p_{i}^{\mathrm{ref}}}{p_{i}^{\mathrm{ref}}}\right)^{2}} \cdot 100 \% \\
& \varepsilon_{Q}^{\mathrm{RMS}}=\sqrt{\frac{1}{N_{t}} \sum_{i=1}^{N_{t}}\left(\frac{Q_{i}-Q_{i}^{\mathrm{ref}}}{\max _{k}\left(Q_{k}^{\mathrm{ref}}\right)}\right)^{2}} \cdot 100 \% \\
& \varepsilon_{p}^{\mathrm{MAX}}=\max _{i}\left|\frac{p_{i}-p_{i}^{\mathrm{ref}}}{p_{i}^{\mathrm{ref}}}\right| \cdot 100 \% \\
& \varepsilon_{Q}^{\mathrm{MAX}}=\max _{i}\left|\frac{Q_{i}-Q_{i}^{\mathrm{ref}}}{\max _{k}\left(Q_{k}^{\mathrm{ref}}\right)}\right| \cdot 100 \% \\
& \varepsilon_{p}^{\mathrm{SYS}}=\frac{\max _{i}\left(p_{i}\right)-\max _{i}\left(p_{i}^{\mathrm{ref}}\right)}{\max _{i}\left(p_{i}^{\mathrm{ref}}\right)} \cdot 100 \% \\
& \varepsilon_{Q}^{\mathrm{SYS}}=\frac{\max _{i}\left(Q_{i}\right)-\max _{i}\left(Q_{i}^{\mathrm{ref}}\right)}{\max _{i}\left(Q_{i}^{\mathrm{ref}}\right)} \cdot 100 \% \\
& \varepsilon_{p}^{\mathrm{DIAS}}=\frac{\min _{i}\left(p_{i}\right)-\min _{i}\left(p_{i}^{\mathrm{ref}}\right)}{\min _{i}\left(p_{i}^{\mathrm{ref}}\right)} \cdot 100 \% \\
& \varepsilon_{Q}^{\text {DIAS }}=\frac{\min _{i}\left(Q_{i}\right)-\min _{i}\left(Q_{i}^{\text {ref }}\right)}{\max _{i}\left(Q_{i}^{\text {ref }}\right)} \cdot 100 \%
\end{aligned}
$$

where superscript "ref" denotes a reference solution at the considered spatial location, subscripts $i$ and $k$ denote time points and $N_{t}$ is the number of these points. In all tests, the integration time in the MOC was sufficiently long to achieve a periodic flow regime and errors are calculated over the last period (the cardiac cycle).

In order to verify implementations of the TLM and the MOC, the linearized model is solved by both methods, and in the next subsection, the obtained results are mutually compared regarding errors as defined above. In the TLM solution, $N$ was 41 (corresponds to the number of harmonics used in the definition of inlet flow rate).

The influence of the convection term is estimated in the second following subsection by comparison of the results from the TLM (linearized model), the MOC (non-linear model) and the experimental data.

Finally, the two methods are compared regarding computational time needed to obtain numerical solution: for the TLM in the frequency domain and for the MOC in the time domain.

\section{Comparison of results from the TLM and the MOC in the case of a 37-artery network model (linearized vs linearized model)}

For the purpose of method verification, the linearized model is solved by the TLM and the MOC. Figure 3 shows the relative difference in two solutions regarding errors defined by equations (28)-(35), in which the TLM solution is the reference one.

\section{Comparison of results from the TLM and the MOC in the case of a 37-artery network model (linearized vs non-linear model)}

The described 37-artery model is solved by the MOC using the non-linear mathematical model (see equations (1)-(4)), and results are compared with the results of the TLM, in which the linearized model was used. Figure 4 shows the relative difference in the two solutions regarding errors defined by equations (28)-(35), in which the TLM solution is the reference one. Table 3 shows the errors of the two solutions with respect to the experimental data at different positions indicated in Figure 2.

\section{Comparison of computational times of the TLM and the MOC}

The computational time of the MOC is nearly proportional to the product of the number of elements $\left(N_{\mathrm{el}}\right)$ 


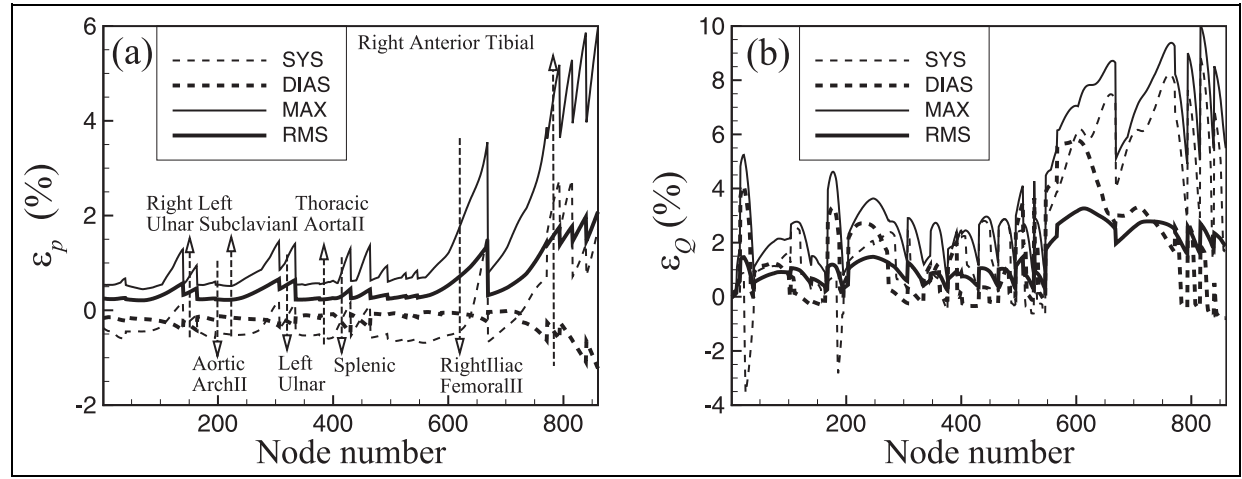

Figure 4. Relative percentage errors of the numerical solution from the non-linear mathematical model obtained by the method of characteristics (MOC) with respect to the solution from the linear model obtained by the transmission line method (TLM):

(a) Errors in pressure: $\varepsilon_{p}^{\mathrm{RMS}}, \varepsilon_{p}^{\mathrm{SYS}}, \varepsilon_{p}^{\mathrm{DIAS}}$ and $\varepsilon_{p}^{\mathrm{MAX}}$ and (b) Errors in flow rate: $\varepsilon_{Q}^{\mathrm{RMS}}, \varepsilon_{Q}^{\mathrm{SYS}}, \varepsilon_{Q}^{\mathrm{DIAS}}$ and $\varepsilon_{Q}^{\mathrm{MAX}}$. Dashed arrows in the left panel indicate positions (node numbers) of measurement sites.

Table 3. Relative percentage errors of the two numerical solutions with respect to the experimental data and relative percentage difference in the two numerical solutions.

\begin{tabular}{|c|c|c|c|c|c|c|c|c|c|}
\hline Arterial segment & Numerical scheme & $\varepsilon_{p}^{\mathrm{RMS}}$ & $\varepsilon_{p}^{\mathrm{MAX}}$ & $\varepsilon_{p}^{\text {SYS }}$ & $\varepsilon_{p}^{\mathrm{DIAS}}$ & $\varepsilon_{Q}^{\mathrm{RMS}}$ & $\varepsilon_{Q}^{\mathrm{MAX}}$ & $\varepsilon_{Q}^{\mathrm{SYS}}$ & $\varepsilon_{Q}^{\text {DIAS }}$ \\
\hline \multirow{3}{*}{ Aortic arch II } & TLM/Exp. & 1.96 & 4.32 & -0.43 & 0.89 & 11.73 & 27.65 & 6.73 & -10.15 \\
\hline & MOC/Exp. & 1.92 & 4.21 & -0.90 & 0.76 & 11.81 & 28.10 & 7.40 & -9.43 \\
\hline & MOC/TLM & 0.23 & 0.54 & -0.47 & -0.13 & 0.39 & 1.03 & 0.63 & 0.67 \\
\hline \multirow[t]{3}{*}{ Thoracic aorta II } & TLM/Exp. & 2.93 & 6.17 & 0.27 & 3.30 & 23.66 & 59.88 & 45.80 & -25.88 \\
\hline & MOC/Exp. & 2.86 & 6.03 & -0.30 & 3.20 & 24.05 & 61.88 & 48.48 & -23.48 \\
\hline & MOC/TLM & 0.23 & 0.57 & -0.56 & -0.10 & 0.81 & 1.89 & 1.84 & 1.63 \\
\hline \multirow[t]{3}{*}{ Left subclavian I } & TLM/Exp. & 2.23 & 4.68 & -3.02 & -2.34 & 13.00 & 29.99 & $-|1.3|$ & $-|1.5|$ \\
\hline & MOC/Exp. & 2.37 & 4.60 & -3.52 & -2.49 & 13.34 & 31.57 & -9.80 & -9.11 \\
\hline & MOC/TLM & 0.22 & 0.51 & -0.51 & -0.15 & 1.34 & 3.15 & 1.70 & 2.70 \\
\hline \multirow[t]{3}{*}{ Right iliac-femoral II } & TLM/Exp. & 2.74 & 5.33 & -1.88 & -1.63 & 19.45 & 40.88 & 33.12 & -24.77 \\
\hline & MOC/Exp. & 3.04 & 6.28 & -2.30 & -1.70 & 21.27 & 50.93 & 40.90 & -17.78 \\
\hline & MOC/TLM & 0.72 & 1.77 & -0.44 & -0.07 & 3.23 & 7.82 & 5.85 & 5.25 \\
\hline \multirow[t]{3}{*}{ Left ulnar } & TLM/Exp. & 2.37 & 4.72 & -1.98 & 0.32 & 11.04 & 18.66 & -2.49 & -10.66 \\
\hline & MOC/Exp. & 2.26 & 4.38 & -2.13 & 0.05 & 11.26 & 18.81 & -1.55 & -10.62 \\
\hline & MOC/TLM & 0.54 & 1.09 & -0.14 & -0.23 & 0.95 & 2.44 & 0.96 & 0.04 \\
\hline \multirow[t]{3}{*}{ Right anterior tibial } & TLM/Exp. & 3.45 & 9.34 & -3.51 & 2.37 & 8.08 & 12.79 & -7.99 & -2.39 \\
\hline & MOC/Exp. & 3.21 & 11.28 & -1.35 & 1.94 & 8.32 & 13.49 & -3.30 & -2.63 \\
\hline & MOC/TLM & 1.56 & 4.43 & 2.24 & -0.42 & 1.96 & 7.02 & 5.10 & -0.26 \\
\hline \multirow[t]{3}{*}{ Right ulnar } & TLM/Exp. & 2.11 & 4.61 & -1.63 & -1.22 & 10.23 & 23.15 & 13.33 & 1.44 \\
\hline & MOC/Exp. & 2.32 & 5.05 & -1.85 & -1.53 & 10.42 & 24.42 & 13.82 & 1.15 \\
\hline & MOC/TLM & 0.39 & 0.73 & -0.22 & -0.32 & 0.56 & $\mathrm{I} .23$ & 0.43 & -0.26 \\
\hline \multirow[t]{3}{*}{ Splenic } & TLM/Exp. & 1.84 & 4.67 & -1.53 & -0.14 & 7.47 & 19.57 & -8.27 & -2.36 \\
\hline & MOC/Exp. & 1.89 & 4.91 & -1.72 & -0.47 & 7.63 & 19.39 & -7.32 & -2.70 \\
\hline & MOC/TLM & 0.30 & 0.81 & -0.19 & 0.26 & 0.65 & 2.25 & 1.03 & -0.34 \\
\hline
\end{tabular}

TLM/Exp.: relative percentage error of the numerical solution from the linear model obtained by the transmission line method (TLM) with respect to the experimental data (Exp.); MOC/Exp.: relative percentage error of the numerical solution from the non-linear model obtained by the method of characteristics (MOC) with respect to the Exp.; MOC/TLM: relative percentage difference of the MOC with respect to the TLM solution.

and the number of integration time steps $N_{\text {int }}=N_{t} N_{\text {per }}$ (where $N_{t}$ is the number of integration time steps within one heart cycle, and $N_{\text {per }}$ is the number of these periods needed to diminish the influence of the initial condition). The computational time needed to find the solution in the frequency domain by the TLM is proportional to the product $N_{\mathrm{el}} N$. The ratio $R_{t}$ of two computational times (the MOC computational time over the TLM time) is proportional to $N_{\text {int }} / N$. Figure 8 shows the variation of $R_{t}$ with $N_{\text {int }} / N$ where circles denote the data obtained from calculations and the line denotes the linear fit through the data. The equation of this linear fit is $R_{t}=10+0.266 N_{\text {int }} / N$ and the square of the correlation coefficient $\left(r^{2}\right)$ of this fit is 0.997 .

\section{Discussion}

\section{Comparison of results from the TLM and the MOC (linearized vs linearized model)}

In the case when the linear model is used in both methods, the same results for pressure and flow rate are 


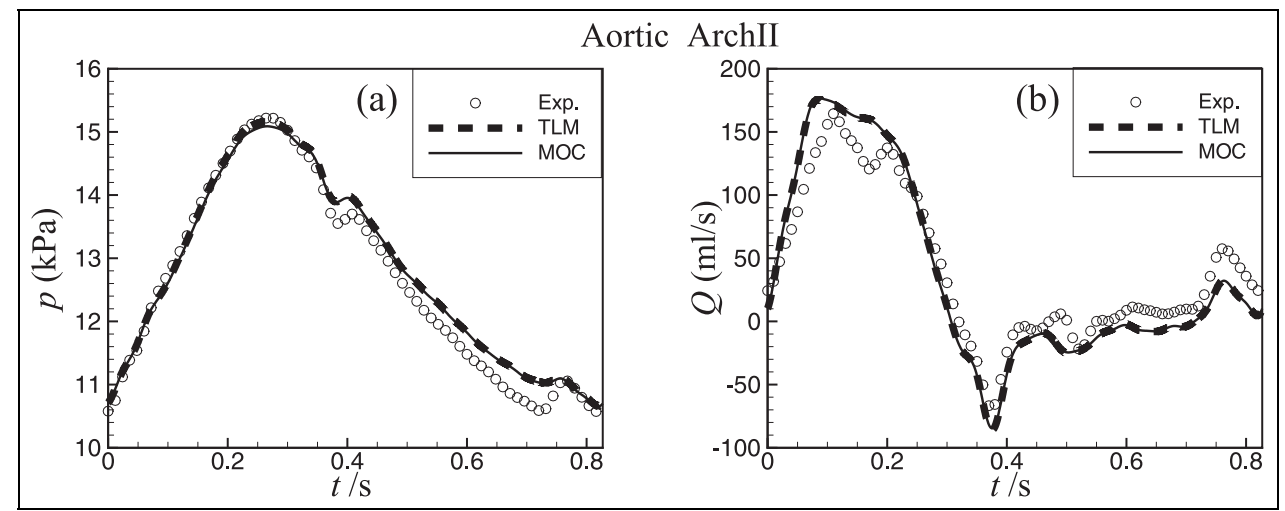

Figure 5. (a) Pressure and (b) flow rate at the midpoint of the Aortic arch II segment. Circles represent in vitro experimental data (Exp.), ${ }^{26}$ dashed lines denote results from the linearized model obtained by the transmission line method (TLM) and solid lines denote results from the non-linear model obtained by the method of characteristics (MOC).

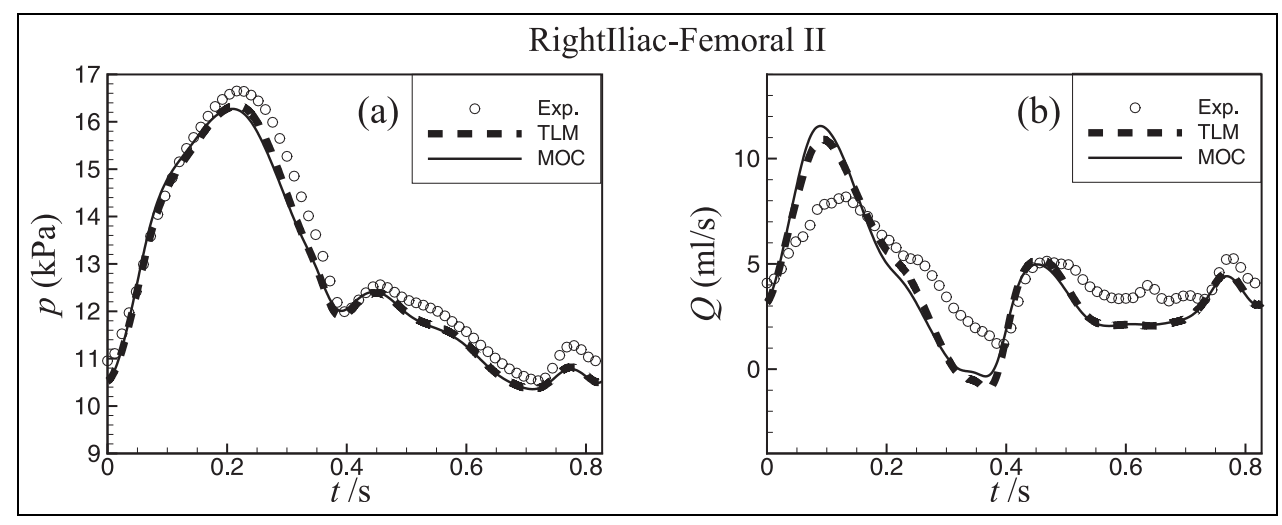

Figure 6. (a) Pressure and (b) flow rate at the midpoint of the Right iliac-femoral II segment. Circles represent in vitro experimental data (Exp.), ${ }^{26}$ dashed lines denote results from the linearized model obtained by the transmission line method (TLM) and solid lines denote results from the non-linear model obtained by the method of characteristics (MOC).

expected. It is visible from Figure 3 that the relative differences in the solutions are below $0.002 \%$ for pressure and below $0.024 \%$ for flow rate. The fact that the obtained solutions from two quite different methods are nearly the same is a good indicator that both methods are properly implemented.

\section{Comparison of results from the TLM and the MOC (linearized vs non-linear model)}

In the case of different models used in the two methods (linearized in the TLM and non-linear in the MOC), a certain level of discrepancy in results is expected. The main reason for the discrepancy is the omission of the convection term in the linearized model. Figure 4 shows the relative difference of the MOC solution with respect to the TLM solution at all nodes. The difference is calculated according to equations (28)-(35) in which the TLM solution is the reference one. The maximal $\varepsilon_{p}^{\mathrm{RMS}}$ is about $2 \%$, and at most nodes this difference is below $0.5 \%$. The maximal $\varepsilon_{Q}^{\mathrm{RMS}}$ is $3.25 \%$, and at most nodes this difference is below $1.5 \%$. The other differences (SYS, DIAS and MAX) are a bit larger, but below $6 \%$ for pressure and $10 \%$ for flow rate. The greatest differences occur at the arterial tree periphery. The applicability of the linearized model can be judged from the results in Table 3. It can be seen that at some measurement sites, the results from the linearized model show smaller discrepancies compared to the experimental data than the results from the non-linear model. In addition, the discrepancy between the results from the linearized and the non-linear model are much smaller than the discrepancy either between the MOC numerical results and experimental data or the TLM numerical results and the experimental data. This is visible in Figures 5-7, which show the results for three selected measurement sites chosen by the criterion of the greatest discrepancy between results from the linearized and the non-linear model. We can conclude that in the considered problem, the non-linear model does not show a significant improvement over the linearized one.

\section{Comparison of computational times of the TLM and the MOC}

The main interest in an arterial blood flow analysis is the steady periodic flow. In the case of time domain methods, the model is integrated from guessed initial 


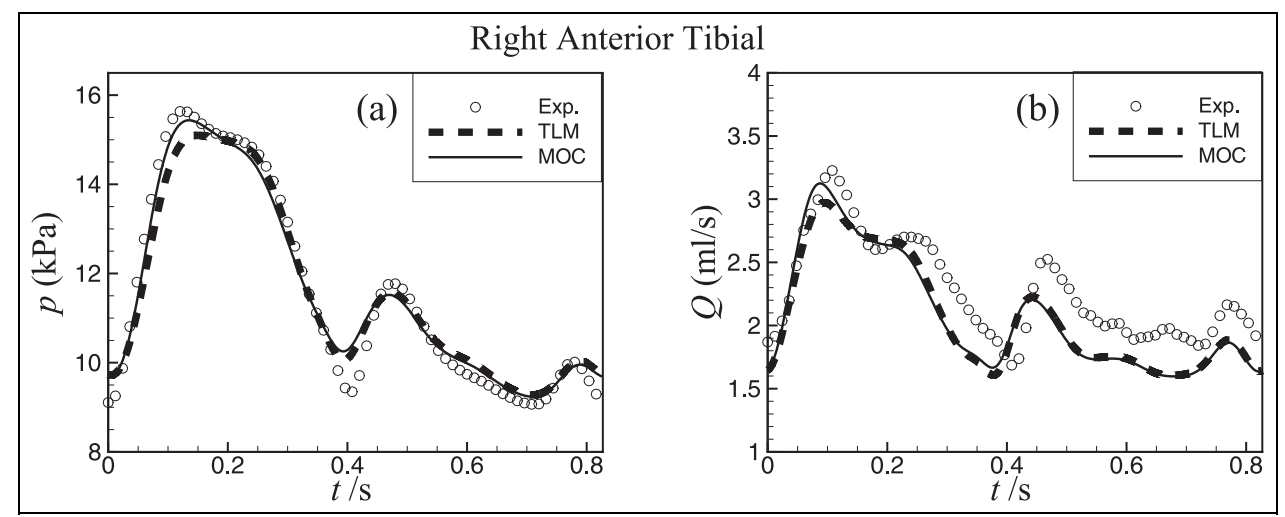

Figure 7. (a) Pressure and (b) flow rate at the midpoint of the Right anterior tibial segment. Circles represent in vitro experimental data (Exp.), ${ }^{26}$ dashed lines denote results from the linearized model obtained by the transmission line method (TLM) and solid lines denote results from the non-linear model obtained by the method of characteristics (MOC).

conditions, so the integration time should be sufficiently long to achieve the periodic solution. When expressed in terms of heart periods, the integration time is $N_{\text {per }} T$, where the minimal value of $N_{\text {per }}$ is needed to diminish initial conditions. $N_{\text {per }}$ ranges from 10 (in the case of a single tube) to 30 (in the case of an arterial network). Experience shows that the pressure and flow rate waves in an arterial tree are properly resolved in the frequency domain by 20 harmonics. Therefore, in the TLM, there is no need to use $N$ greater than 40 . As shown above, the ratio of computational times needed to obtain the solution (in the case of the MOC in the time domain, and in the case the TLM in the frequency domain) by the two methods is proportional to the ratio $N_{\text {int }} / N=N_{\text {per }} N_{t} / N$. If we assume that the ratio $N_{\text {per }} / N$ is nearly equal to one, then $N_{\text {int }} / N$ is proportional to $N_{t}$. For a typical heart period of $T=1 \mathrm{~s}$, and a typical integration time step from 1 to $5 \mathrm{~ms}$, the ratio $N_{\text {int }} / N$ ranges from 200 to 1000 , and according to Figure $8, R_{t}$ ranges from 63 to 276 . Therefore, in typical applications, the TLM is about two orders of magnitude faster than the MOC.

\section{Conclusion}

The proposed semi-analytic TLM for solving the linearized model of blood flow in a viscoelastic arterial tree is simple and easy to implement. In the considered problem, the accuracy of the TLM solution (obtained from the linearized model) with respect to the experimental data is comparable with the accuracy of the MOC results (obtained from the non-linear model). For typical applications, the TLM is at least two orders of magnitude faster than the MOC. Because of that, the TLM could be considered as an alternative method in solving inverse problems or in optimizations where the model needs to be solved repetitively, that is, many times. When solving a linearized model for a given spatial discretization of the arterial tree, the results from the TLM are accurate up to the computer rounding

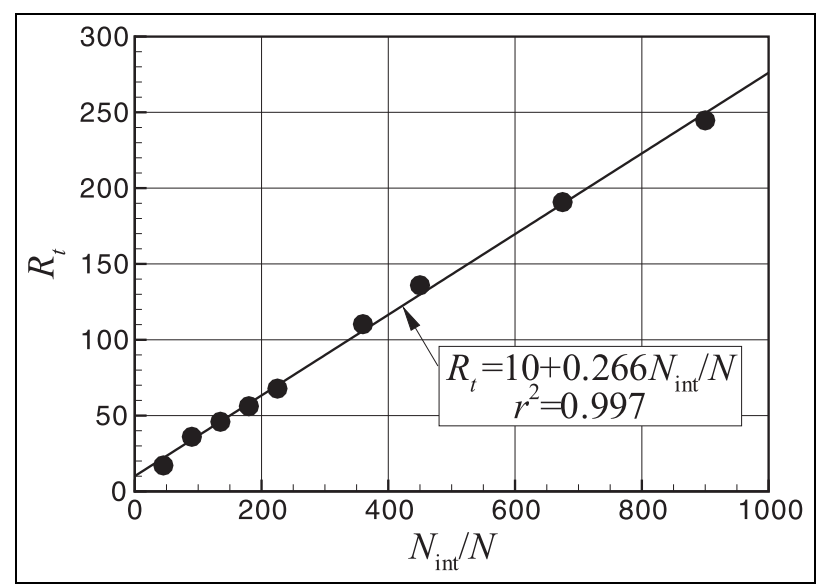

Figure 8. Variation of $R_{t}$ (the computational time of the method of characteristics (MOC) divided by the computational time of the transmission line method (TLM)) with $N_{\text {int }} / N$ (the number of integration time steps in the MOC divided by the number of harmonics in the TLM). Dots denote the data calculated from different cases and the line is the linear fit through these data.

error, and they can serve for the verification of time domain methods.

\section{Declaration of conflicting interests}

The author(s) declared no potential conflicts of interest with respect to the research, authorship and/or publication of this article.

\section{Funding}

The research was funded by the Croatian Ministry of Science, Education and Sports, Grant No. 1201201760-1758.

\section{References}

1. Westerhof N, Lankhaar JW and Westerhof BE. The arterial Windkessel. Med Biol Eng Comput 2009; 47: 131-141. 
2. Azer $\mathrm{K}$ and Peskin CS. A one-dimensional model of blood flow in arteries with friction and convection based on the Womersley velocity profile. Cardiovasc Eng 2007; 7: $51-73$

3. Olufsen MS, Peskin CS, Kim WY, et al. Numerical simulation and experimental validation of blood flow in arteries with structured-tree outflow conditions. Ann Biomed Eng 2000; 28: 1281-1299.

4. Liang FY, Takagi S, Himeno R, et al. Biomechanical characterization of ventricular-arterial coupling during aging: a multi-scale model study. $J$ Biomech 2009; 42: 692-704.

5. Müller LO, Parés C and Toro EF. Well-balanced highorder numerical schemes for one-dimensional blood flow in vessels with varying mechanical properties. $J$ Comput Phys 2013; 242: 53-85.

6. Toro EF and Siviglia A. Flow in collapsible tubes with discontinuous mechanical properties: mathematical model and exact solutions. Commun Comput Phys 2013; 13: 361-385.

7. Alastruey J, Khir AW, Matthys KS, et al. Pulse wave propagation in a model human arterial network: assessment of 1-D visco-elastic simulations against in vitro measurements. J Biomech 2011; 44: 2250-2258.

8. Steele BN, Wan J, Ku JP, et al. In vivo validation of a one-dimensional finite-element method for predicting blood flow in cardiovascular bypass grafts. IEEE Trans Biomed Eng 2003; 50: 649-656.

9. Bessems D, Rutten M and Van De Vosse F. A wave propagation model of blood flow in large vessels using an approximate velocity profile function. $J$ Fluid Mech 2007; 580: 145-168.

10. Watanabe SM, Blanco PJ and Feijóo RA. Mathematical model of blood flow in an anatomically detailed arterial network of the arm. ESAIM Math Model Numer Anal 2013; 47: 961-985.

11. Schaaf BW and Abbrecht PH. Digital computer simulation of human systemic arterial pulse wave transmission: a nonlinear model. J Biomech 1972; 5: 345-364.

12. Wang JJ and Parker KH. Wave propagation in a model of the arterial circulation. $J$ Biomech 2004; 37: 457-470.

13. Acosta S, Puelz C, Rivière B, et al. Numerical method of characteristics for one-dimensional blood flow. J Comput Phys 2015; 294: 96-109.

14. Korade I, Virag Z and Šavar M. Numerical simulation of one-dimensional flow in elastic and viscoelastic branching tube. In: Proceedings of the 6th European conference of computational fluid dynamics (ECFD VI) (eds E Onate, X Oliver and A Huerta), Barcelona, 20-25 July 2014, pp.7124-7131. Barcelona: CIMNE.

15. Snyder MF, Rideout VC and Hillestad RJ. Computer modeling of the human systemic arterial tree. $J$ Biomech 1968; 1: 341-353.

16. McIlroy MB, Seitz WS and Targett RC. A transmission line model of the normal aorta and its branches. Cardiovasc Res 1986; 20: 581-587.

17. Avolio AP. Multi-branched model of the human arterial system. Med Biol Eng Comput 1980; 18: 709-718.

18. Helal MA. Derivation of closed-form expression for the cerebral circulation models. Comput Biol Med 1994; 24: 103-118.

19. Zhang $\mathrm{H}$ and Li JK. A Novel wave reflection model of the human arterial system. Cardiovasc Eng 2009; 9: 39 48.

20. Taylor MG. Wave-travel in a non-uniform transmission line, in relation to pulses in arteries. Phys Med Biol 1965; 10: $539-550$.

21. Roller ML and Clark ME. Precursor cerebral circulation models. J Biomech 1969; 2: 241-250.

22. John LR. Forward electrical transmission line model of the human arterial system. Med Biol Eng Comput 2004; 42: 312-321.

23. Zhu Y, Sprague BJ, Phernetton TM, et al. Transmission line models to simulate the impedance of the uterine vasculature during the ovarian cycle and pregnancy. Eur $J$ Obstet Gynecol Reprod Biol 2009; 144: 184-191.

24. $\mathrm{He} \mathrm{W}$, Xiao $\mathrm{H}$ and Liu X. Numerical simulation of human systemic arterial hemodynamics based on a transmission line model and recursive algorithm. J Mech Med Biol. Epub ahead of print March 2012. DOI: 10.1142/ S0219519411004587.

25. Boileau E, Nithiarasu P, Blanco PJ, et al. A benchmark study of numerical schemes for one-dimensional arterial blood flow modelling. Int J Numer Method Biomed Eng. Epub ahead of print 5 July 2015. DOI: 10.1002/ cnm.2732.

26. Matthys KS, Alastruey J, Peiró J, et al. Pulse wave propagation in a model human arterial network: assessment of $1-\mathrm{D}$ numerical simulations against in vitro measurements. J Biomech 2007; 40: 3476-3486.

27. Smith NP, Pullan AJ and Hunter PJ. An anatomically based model of transient coronary blood flow in the heart. SIAM J Appl Math 2002; 62: 990-1018. 